\title{
Management of gestational hypothyroidism: results of a Brazilian survey
}

\begin{abstract}
Danilo Villagelin', Ana Paula Comarella², Douglas Bernal Tiago ${ }^{3}$, Laura Sterian Ward ${ }^{4}$
\end{abstract}

'Endocrinologia e Metabolismo, Pontifícia Universidade Católica de Campinas (PUC-Campinas). Laboratório de Genética Molecular do Câncer, Faculdade de Ciências Médicas, Universidade Estadual de Campinas (FCM/ Unicamp), Campinas, SP, Brasil 2 Endocrinologia e Metabolismo, Universidade Federal do Espírito Santo (UFES), Vitória, ES. Laboratório de Genética Molecular do Câncer, FCM/ Unicamp, Campinas, SP, Brasil ${ }^{3}$ Ginecologia e Obstetrícia, PUCCampinas, Campinas, SP, Brasil

${ }^{4}$ Laboratório de Genética Molecular do Câncer, FCM/ Unicamp, Campinas, SP, Brasil

\section{Correspondence to:} Danilo Villagelin Hospital e Maternidade Celso Pierro, Endocrinologia e Metabologia

Av. John Boyd Dunlop s/n

13064-904 - Campinas, SP, Brasil dvillagelin@gmail.com

Received on Apr/8/2015

Accepted on May/12/2015

\begin{abstract}
Objectives: Evaluate the management of hypothyroidism in fertile-aged and pregnant women and compare these practices to the recommendations of the Brazilian Society of Endocrinology and Metabolism (SBEM) and the Latin AmericanThyroid Society, published in 2013. Materials and methods: In the first trimester of 2014, SBEM made available to all members an electronic questionnaire based on clinical scenarios in the management of gestational hypothyroidism. The responses of 406 physicians, most of them endocrinologists, were analyzed. Results: Eighty-one per cent of the endocrinologists screen all their pregnant patients for thyroid dysfunction, mostly during the pregestational period or after the first prenatal visit. Following screening, $82 \%$ of the respondents initiate treatment when TSH levels are $>2.5 \mathrm{mIU} / \mathrm{L}$ while $67 \%$ monitor their pregnant patients even if TSH was normal on first trimester screening. For hypothyroid women who are planning pregnancy, $96 \%$ of the clinicians are aware of the importance of adjusting the levothyroxine $\left(\mathrm{LT}_{4}\right)$ dose as soon as pregnancy is confirmed. However, opinions diverge with respect to adjusting the $\mathrm{LT}_{4}$ dose before or after reassessing thyroid function. The most widely used tests for monitoring pregnant women in use of $\mathrm{LT}_{4}$ are TSH and free $\mathrm{T}_{4}(62 \%)$ or TSH alone (21\%). Unanimously, the treatment goal is to achieve the target TSH level for each trimester of gestation. Conclusion: The recommendations of the consensus statements are incorporated into the respondents' clinical practice. It is noteworthy that the great majority of the clinicians favor universal screening. Arch Endocrinol Metab. 2016;60(1):16-20
\end{abstract}

Keywords

Hypothyroidism; pregnant women; surveys

\section{INTRODUCTION}

A normal pregnancy results in a number of important physiological and hormonal changes that modify thyroid function, resulting in greater susceptibility to hypothyroidism in women with reduced thyroidal reserve or iodine deficiency.

Both overt and subclinical hypothyroidism are common in pregnancy. Hypothyroid women are more subject to infertility, have increased prevalence of miscarriage, anemia, gestational hypertension, placental abruption, and postpartum bleeding (1-6). Untreated overt maternal hypothyroidism is associated with adverse events for the neonate: preterm birth, low birth weight, acute respiratory distress syndrome, and neurocognitive deficits (7). Pregnant women with subclinical hypothyroidism seem more prone to preterm delivery and their newborns are more frequently admitted to intensive care (2).
Given the preventable obstetric and neonatal risks involved, recent publications have introduced recommendations for the management of gestational hypothyroidism. In order to determine the impact of those recommendations as well as the most prevalent clinical practices, the Brazilian Society of Endocrinology and Metabolism (Sociedade Brasileira de Endocrinologia e Metabologia - SBEM) made available a survey to its members.

\section{MATERIALS AND METHODS}

An electronic questionnaire survey was sent by e-mail to SBEM members, followed by two reminder e-mails. The survey was based on clinical case scenarios and inquired about the clinical practices related to the management of hypothyroidism during pregnancy.

The survey included questions pertaining to diagnostic evaluation, choice of therapy, and follow-up. Most questions required a single best response to be se- 
lected from multiple choices. Respondents were asked to indicate either a single (10 questions) or multiple (2 questions) answers. All frequencies were adjusted on a $100 \%$ basis excluding the non-respondents.

Survey responses were anonymously collected and stored electronically by the survey service provider.

\section{RESULTS}

\section{Respondent profile}

Our survey included 406 respondents with the following regional distribution: $57 \%$ from the southeast region of Brazil; $18 \%$ from the south; 14\% from the northeast; $8 \%$ from the central-west; and 3\% from the north. Respondent distribution by field of practice was as follows: $89 \%$ endocrinologists, $8 \%$ gynecologists/ obstetricians, $2.5 \%$ general practitioners, and $0.5 \%$ from other specialties. Six respondents did not provide care to fertile-aged or pregnant women and, for that reason, were excluded from the study.

In both public and private Brazilian health care centers, most of the pregnant women with hypothyroidism receive care from an endocrinologist alone (57\%), an endocrinologist with an obstetrician $(39 \%)$, or an obstetrician alone $(4 \%)$.

\section{Screening}

Eighty-one percent out of the endocrinologists chose a universal thyroid dysfunction screening while 13\% advocate selective screening based on risk factors. Another $4 \%$ do not conduct any systematic screening.

The most frequently cited risk factors in the stratification of pregnant women at high risk of thyroid dysfunction are presented in table 1 .

Table 1. Risk factors considered by the respondents in the stratification of pregnant women at high risk for thyroid dysfunction

\begin{tabular}{lc}
\hline \multicolumn{1}{c}{ Risk factor } & $\begin{array}{c}\text { \% } \\
\text { Respondents }\end{array}$ \\
\hline Personal history of thyroid surgery & 100 \\
Personal history of thyroid disease & 97 \\
Personal history of goiter & 97 \\
Family history of thyroid disease in first-degree relatives & 94 \\
History of cervical radiation therapy & 94 \\
History of miscarriage & 94 \\
History of infertility & 88 \\
Family history of autoimmune diseases & 80 \\
Obesity & 38 \\
\hline
\end{tabular}

Different laboratory tests were indicated by the respondents for hypothyroidism screening in pregnant women, as shown in figure 1.

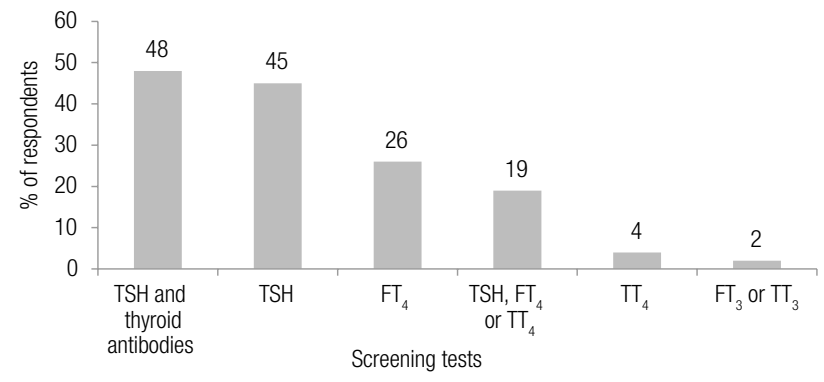

$\mathrm{FT}_{3}$ : tree $\mathrm{T}_{3} ; \mathrm{T}_{3}$ : total $\mathrm{T}_{3} ; \mathrm{FT}_{3}$ : free $\mathrm{T}_{3} ; \mathrm{T}_{3}$ : total $\mathrm{T}_{3} ; \mathrm{FT}_{4}$ : free $\mathrm{T}_{4} ; \mathrm{T}_{4}$ : total $\mathrm{T}_{4} ; \mathrm{TSH}$ : thyroid stimulating hormone.

Figure 1. Most frequent gestational hypothyroidism screening tests chosen by respondents.

The best time point for screening was pointed out as the pregestational visit $(73 \%)$, followed by the first prenatal visit $(24 \%)$. By contrast, $3 \%$ of the respondents reported no specific time.

Most of the physicians (67\%) stated that they follow up their pregnant patients even the ones who had normal TSH levels on the first trimester screening tests. Twenty-six percent out of these physicians remeasure thyroid function for all their pregnant patients in the second and third trimesters of pregnancy; $25 \%$ reassess the thyroid function only in pregnant patients with risk factors; and 16\% measure thyroid antibodies. The remaining $33 \%$ of the surveyed clinicians do not monitor their patients in this setting.

\section{Diagnosis and treatment}

Eighty-two per cent of the respondents consider a TSH level of $>2.5 \mathrm{mIU} / \mathrm{L}$ to initiate treatment of gestational hypothyroidism. For $9 \%$ of those surveyed, the cut-off point is a TSH of $>5 \mathrm{mIU} / \mathrm{L}$, and $7 \%$ adopt the laboratory reference range to guide their management strategy.

The most widely used laboratory tests for monitoring the treatment of pregnant women are reported in figure 2 .

The goal of treatment in gestational hypothyroidism is to maintain TSH concentrations $<2.5 \mathrm{mIU} / \mathrm{L}$ in the first trimester and $<3.0 \mathrm{mIU} / \mathrm{L}$ in the second and third trimesters, according to $94 \%$ of the surveyed physicians.

Considering women with a history of hypothyroidism due to chronic autoimmune thyroiditis, TSH levels of $<2.5 \mathrm{mIU} / \mathrm{L}$ (receiving $\mathrm{LT}_{4}$ ), and planning pregnancy, $96 \%$ of the respondents are aware of the importance of adjusting the dose of $\mathrm{LT}_{4}$ as soon as preg- 
nancy is confirmed. Among these, $48 \%$ increase the $\mathrm{LT}_{4}$ dose before reassessing thyroid function whereas $47 \%$ reassess thyroid function first. For the remaining $4 \%$, it is only necessary to reevaluate thyroid function in the second trimester of gestation.

Most of the physicians monitor their pregnant patients with hypothyroxinemia by measuring TSH and $\mathrm{FT}_{4}$ levels $(35 \%)$ or thyroid antibodies $(28 \%)$ each trimester; $22 \%$ chose $\mathrm{LT}_{4}$ treatment, and $15 \%$ do not follow or treat these patients.

Some clinical scenarios were presented in our survey. The approaches followed by the respondents are described in figure 3 .

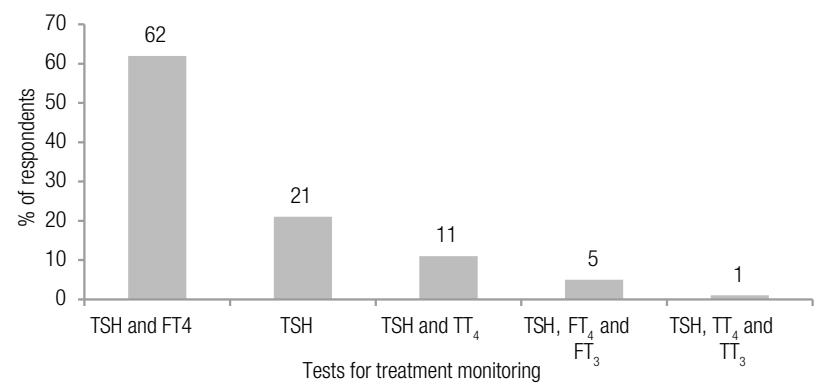

$\mathrm{FT}_{3}$ : free $\mathrm{T}_{3} ; \mathrm{TT}_{3}$ : total $\mathrm{T}_{3} ; \mathrm{FT}_{3}$ : free $\mathrm{T}_{3} ; \mathrm{TT}_{3}$ : total $\mathrm{T}_{3} ; \mathrm{FT}_{4}$ : free $\mathrm{T}_{4} ; \mathrm{TT}_{4}$ : total $\mathrm{T}_{4} ; \mathrm{TSH}$ : thyroid stimulating hormone.

Figure 2. Tests chosen by respondents for thyroid function monitoring of pregnant women treated with LT4.

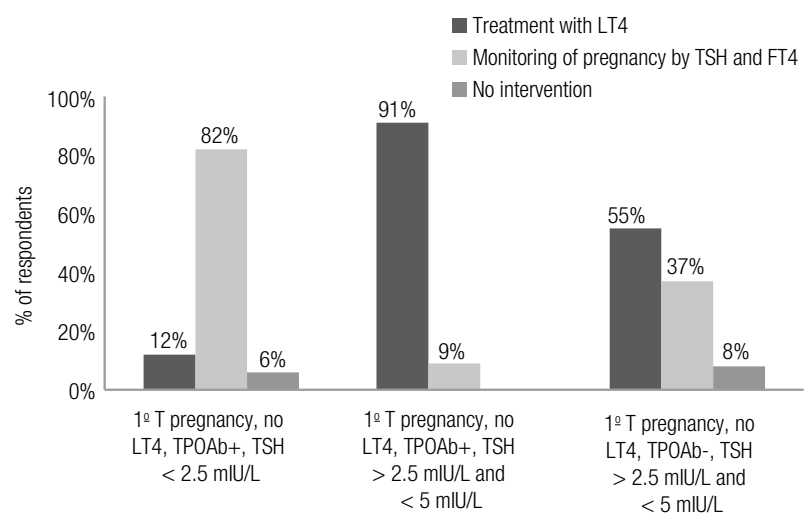

1ำ T: first trimester; TPOAb: anti-thyroid peroxidase antibody

Figure 3. Respondents' clinical approaches to the survey scenarios.

\section{DISCUSSION}

We observed a high degree of agreement between the consensus guidelines and the daily practice of most Brazilian respondents of our electronic survey. However, some aspects are contradictory. In particular, the choice of the majority of endocrinologists for universal screening for thyroid dysfunction. The guidelines recom- mend an active search for high-risk pregnant women, although the evidence to support this recommendation is limited, which justifies the disparity in practices. The leading argument in favor of selective screening based on risk factors is the scarcity of evidence regarding the benefits of using $\mathrm{LT}_{4}$ to treat subclinical gestational hypothyroidism (8). Nevertheless, Dosiou and cols. demonstrated that even when those benefits are lacking, universal screening remains a cost-effective practice (9). In a recent review on this topic, Vila and cols. concluded in favor of universal screening based on the high prevalence of thyroid dysfunction during pregnancy, simple diagnosis, available effective and safe treatment, and the possibility of early intervention, thus reducing the burden of disease (8). It is possible that our endocrinologists were influenced by this literature.

The presence of goiter, or obesity, a personal history of thyroid disease or surgery, cervical radiation therapy, miscarriage, infertility, and a family history of autoimmune or thyroid diseases in first-degree relatives are the risk factors indicated in our survey as well as in the guidelines.

Regarding the laboratory test for screening, there was a slight preference for TSH and thyroid antibodies compared to TSH alone. Thyroid autoimmunity can be found in up to $10 \%$ of pregnant women $(10,11)$ and has a positive association with miscarriage and prematurity $(10,12,13)$. However, this may not be a causal relationship. Furthermore, to date, no studies have been developed evaluating the efficacy of screening by measuring anti-TPO antibodies (TPOAb) (8). The preferred screening test should be sensitive, specific, simple, inexpensive, and widely available (14). These characteristics are consistent with the recommendation of TSH as the screening test of choice provided that trimester-specific reference ranges be applied.

Sixty-seven per cent of the surveyed physicians follow up their pregnant patients with normal TSH on the screening tests by reassessing thyroid function (most of the respondents) or measuring thyroid antibodies. This is an important issue, as demonstrated by Vermiglio and cols. (15), in mildly iodine-deficient regions, were screening performed only during the first trimester of gestation could entail more than $40 \%$ missed diagnoses of hypothyroidism.

The TSH cut-off point of $>2.5 \mathrm{mIU} / \mathrm{L}$ for treatment of gestational hypothyroidism is widely accepted among those surveyed. However, 9\% of them use the reference ranges provided by the laboratories to guide 
their therapeutic management - a fact to be regarded with caution because those ranges may not be adjusted to the population of pregnant women.

Regarding the tests for monitoring treatment, the leading choices were 1) TSH and $\mathrm{FT}_{4}, 2$ ) TSH alone, and 3) TSH and total $\mathrm{T}_{4}\left(\mathrm{TT}_{4}\right)$. Determination of TSH levels is the most sensitive method for diagnosis and monitoring of hypothyroidism. Measuring $\mathrm{FT}_{4}$ can also be helpful, but the most common determination methods are influenced by the low serum albumin levels and high TBG levels typical of pregnancy. Alternatively, $\mathrm{TT}_{4}$ could be determined and adjusted by multiplying its range by 1.5 -fold (14).

Thyroxine requirements increase at the initial stages of pregnancy, and this need is progressive up until 16 to 20 weeks of gestation (7). Therefore, hypothyroid pregnant women who are already on $\mathrm{LT}_{4}$ replacement will typically require an increase in the preconception $\mathrm{LT}_{4}$ dose. According to the guidelines, pregestational TSH levels should ideally be maintained $<2.5 \mathrm{mIU} / \mathrm{L}$ (7,14,16-18). However, newly pregnant hypothyroid women without a previous TSH determination or immediate access to their physician should increase their daily dose of $\mathrm{LT}_{4}$ by $30 \%$ or include 2 additional $\mathrm{LT}_{4}$ tablets in their weekly dosage $(14,18)$. If TSH is first measured after pregnancy is confirmed, $\mathrm{LT}_{4}$ dosage can be increased on the basis of the variation in TSH levels (7). In our survey, $47 \%$ of the respondents indicated that they reassess thyroid function as soon as pregnancy is confirmed while $48 \%$ augment the dose of $\mathrm{LT}_{4}$ after pregnancy confirmation but before remeasuring thyroid function. It is likely that access to health care services have an influence on the chosen course of action.

Maternal hypothyroxinemia is defined as normal TSH concentrations with $\mathrm{FT}_{4}$ levels below the $5^{\text {th }}$ percentile for the reference range. The current consensus recommends that hypothyroxinemia should not be treated (18), since $\mathrm{FT}_{4}$ measurements can be inconsistent during pregnancy and the evidence of the impact of this condition on the neuropsychological development of the offspring is scarce (19). Nevertheless, approximately $22 \%$ of those surveyed would treat this clinical condition.

A number of clinical scenarios were proposed in our survey. In the case of pregnant women with subclinical hypothyroidism diagnosed in the first trimester, negative for TPOAb, with no previous history of $\mathrm{LT}_{4}$ replacement, $55 \%$ of the clinicians choose treatment while
$37 \%$ would monitor these women by TSH and $\mathrm{FT}_{4}$ measurements in each trimester of gestation. The consensus statements admit the treatment of this condition, considering its potential benefits and low risk, but the data are insufficient to support a recommendation $(14,17)$. Thus, the choice of more than one-third of those surveyed is also justified. In the setting of subclinical gestational hypothyroidism (without previous $\mathrm{LT}_{4}$ treatment) and positive TPOAb, the vast majority of the practitioners initiate replacement with $\mathrm{LT}_{4}$, which is in agreement with the guidelines $(14,18)$. For pregnant women with no previous use of $\mathrm{LT}_{4}$, euthyroid on the screening test, yet TPOAb-positive, $82 \%$ of the surveyed physicians maintain follow-up with TSH and $\mathrm{FT}_{4}$ determinations. The consensus statements corroborate this approach, to be conducted every 4 weeks up to mid-pregnancy and at least one more time between 26 and 32 weeks of gestation (18).

Some clinical issues addressed in our survey were largely consistent with the current recommendations: the choice of pregestational or first prenatal visit for screening (7), the cut point of TSH $>2.5 \mathrm{mIU} / \mathrm{L}$ for diagnosis and treatment of hypothyroidism during pregnancy, and the goal of maintaining TSH levels within the target range for each trimester of gestation when in use of $\operatorname{LT}_{4}(14,17,18)$.

\section{CONCLUSION}

Consensus statements are important tools in modern medicine and have been incorporated into the daily practice of the respondents. However a few controversial aspects persist, such as universal screening versus aggressive case finding.

Disclosure: no potential conflict of interest relevant to this article was reported.

\section{REFERENCES}

1. Abalovich M, Gutierrez S, Alcaraz G, Maccallini G, Garcia A, Levalle $O$. Overt and subclinical hypothyroidism complicating pregnancy. Thyroid. 2002;12(1):63-8.

2. Casey BM, Dashe JS, Wells CE, McIntire DD, Byrd W, Leveno KJ, et al. Subclinical hypothyroidism and pregnancy outcomes. Obstet Gynecol. 2005;105(2):239-45.

3. Glinoer D, Soto MF, Bourdoux P, Lejeune B, Delange F, Lemone $M$, et al. Pregnancy in patients with mild thyroid abnormalities: maternal and neonatal repercussions. J Clin Endocrinol Metab. 1991;73(2):421-7. 
4. Leung AS, Millar LK, Koonings PP, Montoro M, Mestman JH. Perinatal outcome in hypothyroid pregnancies. Obstet Gynecol. 1993;81(3):349-53.

5. Man EB, Brown JF, Serunian SA. Maternal hypothyroxinemia: psychoneurological deficits of progeny. Ann Clin Lab Sci. $1991 ; 21(4): 227-39$

6. Haddow JE, Palomaki GE, Allan WC, Williams JR, Knight GJ, Gagnon J, et al. Maternal thyroid deficiency during pregnancy and subsequent neuropsychological development of the child. N Engl J Med. 1999;341(8):549-55.

7. De Groot L, Abalovich M, Alexander EK, Amino N, Barbour L, Cobin $\mathrm{RH}$, et al. Management of thyroid dysfunction during pregnancy and postpartum: an Endocrine Society clinical practice guideline. J Clin Endocrinol Metab. 2012;97(8):2543-65.

8. Vila L, Velasco I, González S, Morales F, Sánchez E, Torrejón S, et al. Controversies in endocrinology: On the need for universal thyroid screening in pregnant women. Eur J Endocrinol. 2013;170(1):R17-30.

9. Dosiou C, Barnes J, Schwartz A, Negro R, Crapo L, StagnaroGreen A. Cost-effectiveness of universal and risk-based screening for autoimmune thyroid disease in pregnant women. J Clin Endocrinol Metab. 2012;97(5):1536-46.

10. Jaén Díaz JI, López De Castro F, Cordero García B, Santillana Balduz F, Sastre Marcos J, Martín Dal Gesso C. [Incidence of postpartum thyroiditis and study of possible associated factors]. Med Clin (Barc). 2009;132(15):569-73.

11. Toulis KA, Goulis DG, Venetis CA, Kolibianakis EM, Negro R, Tarlatzis BC, et al. Risk of spontaneous miscarriage in euthyroid women with thyroid autoimmunity undergoing IVF: a meta-analysis. Eur J Endocrinol. 2010;162(4):643-52.
12. Negro R. Thyroid autoimmunity and pre-term delivery: brief review and meta-analysis. J Endocrinol Invest. 2011;34(2):155-8.

13. Prummel MF, Wiersinga WM. Thyroid autoimmunity and miscarriage. Eur J Endocrinol. 2004;150(6):751-5.

14. Brenta G, Vaisman M, Sgarbi JA, Bergoglio LM, Andrada NC, Bravo $P$, et al. Clinical practice guidelines for the management of hypothyroidism. Arq Bras Endocrinol Metab. 2013;57(4):265-91.

15. Vermiglio F, Lo Presti VP, Castagna MG, Violi MA, Moleti M, Finocchiaro MD, et al. Increased risk of maternal thyroid failure with pregnancy progression in an iodine deficient area with major iodine deficiency disorders. Thyroid. 1999;9(1):19-24.

16. Garber JR, Cobin RH, Gharib H, Hennessey JV, Klein I, Mechanick JI, et al. Clinical practice guidelines for hypothyroidism in adults: cosponsored by the American Association of Clinical Endocrinologists and the AmericanThyroid Association. Endocr Pract. 2012;18(6):988-1028.

17. Sgarbi JA, Teixeira PF, Maciel LM, Mazeto GM, Vaisman M, Montenegro Junior RM, et al. The Brazilian consensus for the clinical approach and treatment of subclinical hypothyroidism in adults: recommendations of the thyroid Department of the Brazilian Society of Endocrinology and Metabolism. Arq Bras Endocrinol Metabol. 2013;57(3):166-83.

18. Stagnaro-Green A, Abalovich M, Alexander E, Azizi F, Mestman J, Negro R, et al. Guidelines of the American Thyroid Association for the diagnosis and management of thyroid disease during pregnancy and postpartum. Thyroid. 2011;21(10):1081-125.

19. Vaidya B, Hubalewska-Dydejczyk A, Laurberg P, Negro R, Vermiglio F, Poppe K. Treatment and screening of hypothyroidism in pregnancy: results of a European survey. Eur $\mathrm{J}$ Endocrinol. 2012;166(1):49-54. 